\section{MONITORING MOVEMENTS WITH HIGH TRUNK ACCELERATION DURING BADMINTON GAMES: AN APPROACH COMBINING A MICROSENSOR UNIT AND VIDEO ANALYSIS}

'Shogo Sasaki, ${ }^{2}$ Yasuharu Nagano, ${ }^{1}$ Yui Shimada, ${ }^{3}$ Hiroshi Ichikawa. ${ }^{1}$ Tokyo Ariake University of Medical and Health Sciences, Tokyo, Japan; ${ }^{2} J a p a n$ Women's College of Physical Education, Tokyo, Japan; ${ }^{3}$ Niigata University of Health and Welfare, Niigata, Japan

10.1136/bjsports-2021-IOC.188

Background Stiff landing is associated with increased anterior cruciate ligament (ACL) injury risk, especially in young female athletes. As an alternative to a force platform, a tri-axial accelerometer is a useful tool to reflect the magnitude of the ground reaction force exerted during games.

Objective To clarify the movements that require high trunk accelerations and their frequency during badminton games.

Design Observational study.

Setting Youth athletes, local tournament levels.

Participants Forty-five female badminton players [17 junior high school (JHS) and 28 high school (HS) athletes].

Assessment of Risk Factors Movements that generated $>4 \mathrm{G}$ resultant acceleration were assessed as a risk for ACL injury.

Main Outcome Measurements Frequency and characteristics of the movements that generated $>4 \mathrm{G}$ acceleration during singles games of badminton.

Results A total of 6,306 movements generated $>4 \mathrm{G}$ acceleration during an 896-min game duration (7.04 cases/min; 95\% confidence interval (CI), 6.87-7.21 cases/min). HS players (7.27 cases/min; 95\% CI, 7.05-7.48 cases/min) had a higher incidence of great trunk acceleration compared with JHS players (6.58 cases/min; 95\% CI, 6.29-6.87 cases/min). The top three most frequent movements were landing after an overhead stroke (JHS, 1.13 cases/min; HS, 1.58 cases/min), lunging during an under-/side-hand stroke (JHS, 0.98 cases/min; HS, 1.27 cases/min), and cutting from a split stepping (JHS, 0.96 cases/min; HS, 1.29 cases/min).

Conclusions HS athletes had an opportunity to incur exposure to high-loading movements during badminton games, which supports an epidemiological survey's results that the incidence rate of ACL injury in HS athletes is six times higher than that in JHS athletes. In addition, previously reported mechanisms of ACL injury in badminton (i.e. single-leg landing after an overhead stroke and plant-and-cut manoeuvre after a lunge stepping) were revealed as the high-frequency movements that generated $>4 \mathrm{G}$ acceleration. This study suggests that video analysis with micro-sensor technology can individually detect the high-loading movements during game-play situations, which contribute to identifying the high-risk athletes from the on-court/field perspective.

\section{ABSTRACT WITHDRAWN}

\section{A DYNAMIC WARM UP PROGRAMME REDUCES KNEE AND ANKLE INJURIES AMONG YOUTH MALE SUB-ELITE FOOTBALL PLAYERS}

Mojtaba Asgari, Thomas Jaitner. Institue for Sport and Sports Science, TU Dortmund University, Dortmund, Germany

10.1136/bjsports-2021-IOC. 189
Background Dynamic exercises appear to be more practical in preventing football-related injuries.

Objective To evaluate effects of a dynamic warm up programme including dynamic stretch, strength and plyometric exercises (DSP) on prevention of injuries.

Design Prospective non-randomized controlled trial.

Setting Competitive level.

Interventions Two volunteer teams of Iranian U21 league were assigned to control $(\mathrm{n}=29,18.5 \pm 1.2)$ and DSP $(\mathrm{n}=25,18.7$ \pm 1.5 ) groups. Over a football season (2018), the DSP performed the specific warm up lasting $15 \mathrm{~min}$ three times weekly with a progression of intensities over time. The control group followed its routine warm up including football drills and physical warm ups without a specific prevention approach. Injuries and time exposure were recorded according to the FIFA consensus statement. Data were analysed by ChiSquare Test while the level of significant was set as $\mathrm{p} \leq 0.05$.

Main Outcome Measurements Injury incidence and risk ratio for ankle and knee.

Results In total, $7020 \mathrm{~h}$ of training and competition and 37 lower extremity injuries were recorded; about $65 \%$ in the control group. The injury incidence for DSP and control were 1.8 and 3.4 respectively. Incidences of knee and ankle injuries for DSP and control were 1.0, 0.9, 2.3 and 1.3, respectively. The risk ratio for knee and ankle injuries was higher in the control group by 2.3 and 1.5 times, respectively. Chi-square testing indicated a significantly lower knee injury incidence for the DSP group $(p=0.03)$. Despite a $30 \%$ lower injury incidence, the difference of ankle injuries between the groups was not significant $(\mathrm{p}=0.52)$.

Conclusions Applying DSP as regular warm up significantly reduced the incidence of knee injuries in youth footballers, which is assumed to be due to a better stimulation of the control mechanisms of the postural system followed by performing the programme. For the ankle, exercises that are more specific should be applied though the limited small sample size may also affect the results.

\section{EFFECTS OF THE FIFA 11+ INJURY PREVENTION PROGRAMME ON PHYSICAL AND TECHNICAL PERFORMANCE, BIOMECHANICAL MEASURES AND PHYSIOLOGICAL RESPONSES}

${ }^{1}$ Mojtaba Asgari, ${ }^{1}$ Thomas Jaitner, ${ }^{2}$ Bahareh Nazari. 'Institue for Sport and Sports Science, TU Dortmund University, Dortmund, Germany: ${ }^{2}$ Sport Medicine department, Faculty of Physical Education and Sports Science, University of Tehran, Tehran, Iran (Islamic Republic of)

\subsection{6/bjsports-2021-IOC.190}

Background Performance aspects of the FIFA11+ programme have not been generally reviewed.

Objective To synthetize the literature on the effects of the FIFA $11+$ on the performance

Setting Systematic review

Participants Football players

Search procedure We searched five online databases for the period from 2006 to May. 2021, using five predefined keywords in conjunction to sub-keywords. Totally, 461 potential references recorded through Endnote and imported. Out of the 117 potential titles and abstracts screened by two independent researchers through Covidence, 54 full-text assessed for eligibility of which, 28 were included. Quality of studies and risk of bias were then assessed. 